Research Article

\title{
Time Allocation and Optimization in Time-Reversal Wireless Powered Communication Networks
}

\author{
Fangwei Li, ${ }^{1}$ Yue Wu $\mathbb{D}^{1},{ }^{1}$ Yifang $\mathrm{Nie}^{2}$ and Ce Shi ${ }^{1}{ }^{1}$ \\ ${ }^{1}$ Chongqing Key Laboratory of Mobile Communications Technology, \\ Engineering Research Center of Mobile Communications of the Ministry of Education, \\ School of Communication and Information Engineering, Chongqing University of Posts and Telecommunications, \\ Chongqing 400065, China \\ ${ }^{2}$ Chongqing Technology and Business University, Chongqing 400065, China
}

Correspondence should be addressed to Yue Wu; s170131220@stu.cqupt.edu.cn

Received 22 September 2019; Revised 10 May 2020; Accepted 20 May 2020; Published 22 July 2020

Academic Editor: Hervé Aubert

Copyright (c) 2020 Fangwei Li et al. This is an open access article distributed under the Creative Commons Attribution License, which permits unrestricted use, distribution, and reproduction in any medium, provided the original work is properly cited.

This paper studies optimal resource allocation in the wireless powered communication networks (WPCN) combined with time reversal (TR) in which one hybrid access point (H-AP) broadcasts constant wireless energy to a set of distributed users in the downlink (DL) and receives information from the users via space division multiple access (SDMA) in the uplink (UL). Inevitable interferences will occur when users transmit information in the UL simultaneously and the special space-time focusing of TR is used to suppress the interferences. An efficient protocol is proposed to support wireless energy transfer (WET) and TR in the DL and wireless information transmission in the UL for the proposed TR-WPCN. We optimize the time allocations to the H-AP for DL WET, DL TR, and UL WIT to maximize the sum throughput. Due to the nonconvexity of the studied optimization problem, we optimize variables successively, where the nonconvex optimization problem is transformed into the convex optimization problem. The approximate convex optimization problem can then be solved iteratively combined with the dichotomy method. Simulation results show that the proposed scheme can effectively suppress interferences and improve system performance.

\section{Introduction}

With the rapid development of wireless communication network and the rapid growth of the number of wireless terminals access to the network, a large number of Internet of Things (IoT) devices represented by sensor nodes will exist in future wireless communication systems. Thus, how to provide stable and continuous power supply for these ubiquitous devices is an urgent problem to be solved for IoT. Radiofrequency (RF) energy harvesting (EH) has recently emerged as a promising solution to unlimited power supply for wireless networks. Simultaneous wireless information and power transfer (SWIPT) $[1,2]$ and WPCN $[3,4]$ are the main methodologies for $\mathrm{EH}$ in wireless network.

In SWIPT, the base station can simultaneously transmit information and energy to the receivers. Power splittingbased SWIPT was discussed in [1], and the decoding cost at the relay was proved less than the destination. A general time switching protocol for the SWIPT broadcast channels was studied in [2], and the achievable rate region was analyzed under EH constraint at the receivers. Compared with SWIPT, all the received RF signals are used to provide power supply in WPCN, so the users can receive more energy and the transmission distance is farther and wider. Besides, the uplink transmission is considered in WPCN which is more compatible with the sensor network that needs to upload detection information regularly.

In WPCN, the wireless communication devices receive RF signal in the DL and store the energy which is converted by the RF signal in the rechargeable batteries. Thus, the devices can communicate with each other and send data to the H-AP in the UL. There are two access approaches in the WPCN system: time division multiple access (TDMA) and SDMA. 
TDMA is the main access method discussed in WPCN which can effectively avoid interference between users [5-9]. A time allocation algorithm was proposed in [5] to maximize the common throughput of users via TDMA, and a "double near and far" problem was found in the multiuser WPCN system. In [6], a weighted throughput optimization problem was discussed for the two-node WPCN where energy can be purposely transferred from an energy-rich network node (e.g., an access point) to terminal devices. The work in [7] considered two different scenarios whether PS and the sensor nodes belong to the same service operator and two optimal solutions are proposed to maximize the system sum throughput of the sensor network for the two scenarios. WPCN with heterogeneous nodes (nodes with and without energy harvesting capabilities) was studied, and the optimal solutions were proposed in $[8,9]$ to maximizing the sum throughput or maximizing the minimum throughput. Abad and Ercetin [10] studied a sensing resolution optimization problem for a wireless powered device which is powered by wireless power transfer from an access point. And a balance between the quality of the sensed data and the probability of successfully delivering it was struck. Full-duplex WPCN was studied in [11-13]. Ju and Zhang [11] considered both cases with perfect and imperfect self-interference cancellation (SIC) at the H-AP aiming at maximizing the users' weighted sum rate of UL information transmission. Kang et al. [12] investigated the sum-throughput maximization problem and the total time minimization problem for the proposed full-duplex wireless powered communication network. AbdElmagid et al. [13] departed from the strong assumption adopted in $[11,12]$, where the energy harvested in a slot is used completely in that slot, and embraced a long-term optimization framework to maximize the long-term weighted throughput.

Different from TDMA which can effectively avoid interference between users in the UL, SDMA can further improve the system throughput with the existence of interference. SDMA was mentioned in [14] which can further improve the system throughput in the case of increased interference. Nonorthogonal multiple access (NOMA) was introduced in WPCN in $[15,16]$. Users transmit information via SDMA in the UL to enhance the power-bandwidth efficiency. Abd-Elmagid et al. [15] considered a trade-off between maximizing the sum throughout and achieving fairness through maximizing the minimum throughput in NOMA-WPCN. Aboelwafa et al. [16] studied a generic scenario, in which the energy rich and access point do not coincide in WPCN with NOMA to optimize the sum throughput. However, the interference between users is not carefully considered in the UL. In this paper, we introduce TR in WPCN to focusing signals and suppressing the uplink transmission interference. In [17], a "tunneling effect" caused by TR was verified in the cloud radio access network (C-RAN) which can improve the uplink throughput. Tran et al. [18] outlined the specific applications of TR in green IoT, especially in the energy harvesting system, indicating that TR was a promising research direction of IoT. Chen et al. [19] introduced TR to the energy harvesting system with an active eavesdropper to reduce the system error rate.
Our contributions can be summarized as follows. We consider a WPCN composed of an H-AP and $N$ users $(N \geq 2)$. As $\mathrm{H}-\mathrm{AP}$ receives information from the users via SDMA in the UL, inevitable interferences will occur. TR is introduced in WPCN to suppress interference and improve the system performance. And a novel protocol is proposed to support WET and TR in the DL and wireless information transmission in the UL for the proposed TR-WPCN. We optimize the time allocations to the H-AP for DL WET, DL TR, and UL WIT to maximize the sum throughput. Since the optimization problem is nonconvex, we optimize variables successively, where the nonconvex optimization problem is transformed into the convex optimization problem. Our numerical results show the superiority of the proposed TRWPCN.

The rest of this paper is organized as follows. A novel protocol of the TR-WPCN system is proposed in Section 2, adding a part to transmit probe signal. In Section 3, we labor the three parts of the TR-WPCN system and obtain its performance parameters. In Section 4, we obtain the optimum time allocation scheme to maximize the system throughput. Section 5 provides simulation results. Finally, conclusions are drawn in Section 6.

\section{System Model}

We study a multiuser WPCN system composed of one H-AP and $N$ users. It is assumed that the H-AP and all users are equipped with one single antenna each. The H-AP supports wireless transmission and can also send and receive information. Users can convert the obtained RF signals into energy and store it in the rechargeable batteries; then, they transfer information uplink using the stored energy in the downlink (Figure 1).

To enhance the spectral efficiency, we assume that all users transmit information to the H-AP via SDMA after receiving the probe signal. At this point, we propose a novel protocol in Figure 2 which is different from the traditional harvest-then-transmit protocol.

Corresponding to the three phases in Figure 2, the system has divided a block transmission time into three parts: DL wireless energy transmission phase, TR phase, and the UL wireless information transmission phase. The first $\tau^{D}$ amount of time is assigned to the DL for the users to obtain energy from the RF signals sent by the H-AP and store it in the rechargeable batteries. Then, $\tau^{\text {tr }}$ represents the time portion in each block allocated to users to receive the timereversal probe signal sent by the H-AP. The last $\tau^{U}$ amount of time is assigned to the UL for the users to transmit information to H-AP. The length of each block transmission time can be different from block to block; however, for convenience, we assume a normalized unit block time $T=1$ in the sequel without loss of generality.

\section{Proposed WPCN System}

3.1. Downlink Energy Transmission. During the DL wireless energy transmission, the DL channel from the H-AP to the $i$ th user is denoted by $h_{i} \in \mathbb{C}^{L}$. Assuming all the channels 


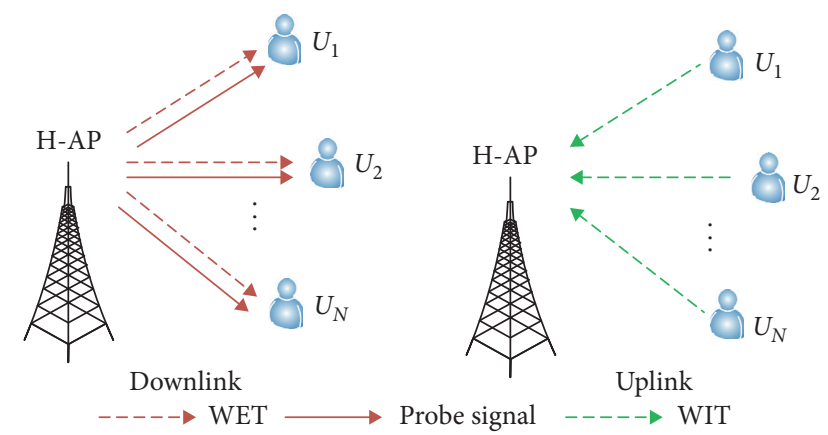

Figure 1: System model.

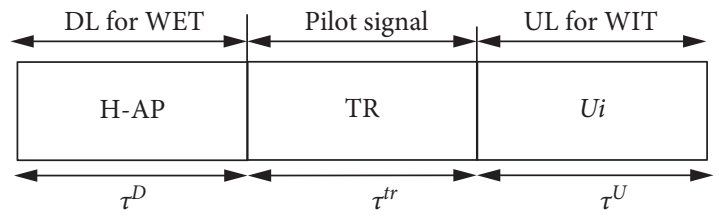

FIgURE 2: The proposed protocol.

follow independent quasistatic flat fading, so the $h_{i}$ remains constant during one block transmission time. The downlink RF energy signal sent by the H-AP is denoted by $x_{A}$, and $x_{A}$ is an arbitrary complex random signal satisfying $E\left[\left|x_{A}\right|^{2}\right]=P_{A}$, where $P_{A}$ denotes the transmit power at $\mathrm{H}$-AP. So the energy signal received by the user is given by

$$
y_{i}=\left(\frac{1}{d_{i}}\right)^{\gamma_{i}} h_{i} x_{A}+z_{i}, \quad i=1, \epsilon, \ldots, N,
$$

where $d_{i}$ indicates the distance from the $i$-th user to the $\mathrm{H}$-AP and $\gamma_{i}$ is the path loss factor. For the convenience of the following description, let $\widetilde{h}_{i}=\left(1 / d_{i}\right)^{\gamma_{i}} h_{i}$ and $z_{i}$ indicates that the additive white Gaussian noise received by the $i$-th user with an average value of 0 and the variance is $\sigma^{2}$.

Therefore, the DL transmission energy received by each user is given by

$$
E_{i}=\xi_{i} P_{A}\left\|\widetilde{h}_{i}\right\|^{2} \tau^{D}, \quad i=1, \ldots, N,
$$

where $\xi_{i}$ is the energy harvesting efficiency for each user, where $0<\xi_{i}<1, i=1, \ldots, N$, in the subsequent text, let $\xi_{1}=\cdots=\xi_{N}=\xi=1$ to facilitate the calculation.

3.2. Time Reversal. TR is a digital signal processing technology that utilizes probe signals to obtain channel state information (CSI) so that the source signal can be perfectly reconstructed in its original position. The operation process of TR can be divided into two steps, including the probe phase and the reversal phase. During the probe phase, the receiver first sends the TR probe pulse signal $p(t)$ which contains CSI to the sender:

$$
y(t)=p(t) \otimes h(t)
$$

where $\otimes$ represents convolution and $h(t)$ is the channel impulse response (CIR) from the receiver to the sender:

$$
h(t)=\sum_{l=0}^{L-1} \alpha_{l} \delta\left(t-\tau_{l}\right)
$$

Since it is in a multipath environment, CSI is composed of multiple paths. $\alpha_{l}$ and $\tau_{l}$ represent the attenuation amplitude and delay factor of the $l-$ th path, respectively. $\delta_{l}$ is the Dirichlet function and $L$ represents the total number of nonzero multipaths.

In the reversal phase, after receiving the probe pulse signal, the sender performs time-reversal processing on the received signal $y(t)$ to obtain a TR signal:

$$
y(-t)=p(-t) \otimes g(t)
$$

where $g(t)$ is the normalized TR channel signature. After that, the sender sends the TR signal together with the signal to be sent $x(t)$ to the receiver via the original path. The signals received at the receiver are as follows:

$$
\begin{aligned}
y^{\prime}(t) & =x(t) \otimes y(-t) \otimes h(t) \\
& =x(t) \otimes p(-t) \otimes g(t) \otimes h(t)
\end{aligned}
$$

When users simultaneously transmit information via SDMA in the UL, interuser interference (IUI) occurs. Take into account the influence of parameters such as intersymbol interference (ISI), path loss, and channel state on the received signal quality during transmission, timereversal technique is added to the system transmitting end [20-22]. Utilizing the unique space-time focusing characteristics of time reversal in the multipath scattering environment, the multiuser interference and cochannel interference in the system can be resisted. After receiving the RF energy signal, the UE transmits the probe signal of the time-reversal period to obtain the CIR. According to channel reciprocity, the equivalent channel response can be obtained. When the users transmit information uplink, the conjugate inversion form of the equivalent channel response will be generated and this will cause a "tunneling effect" in a multipath environment [17]. Due to the "tunneling effect" of TR, each user transmits the signals independently in their respective paths, which effectively suppresses interference. The signal signature after TR is as follows:

$$
g_{i}[l]=\frac{\tilde{h}_{i}^{*}[L-1-l]}{\sum_{l=0}^{L-1}\left\|\widetilde{h}_{i}(l)\right\|^{2}},
$$

where $*$ represents conjugate, $L$ is the total number of multipath, and $g_{i} \in \mathbb{C}^{L}$.

3.3. Uplink Information Transmission. During the UL wireless information transmission, the signal H-AP received from the user is as follows:

$$
y_{U_{i}}=\left(\widetilde{h}_{i} \otimes g_{i}\right)[L] x_{i}+z_{U_{i}}, \quad i=1, \ldots, N .
$$

Assuming that the $i$-th user's capacity usage ratio is $\eta_{i}$, then, the energy used to transmit the information is $\eta_{i} E_{i}$, and the transmission power of $U_{i}$ is given by 


$$
P_{i}=\frac{\eta_{i} E_{i}}{\tau^{U}}, \quad i=1, \ldots, N
$$

Because the user's capacity usage ratio is not the focus of this paper, let $\eta_{1}=\cdots=\eta_{i}=\eta$ in the following for convenience. Here, we consider a linear energy harvesting model instead of a practical nonlinear one because the nonlinear energy harvest model is not easy to construct and the real data are not easy to capture. Both linear energy harvesting model and nonlinear energy harvesting model are the function of $P_{A} q_{i}$, where $P_{A}$ denotes the transmit power at $\mathrm{H}$-AP and $q_{i}$ is the channel power gain between the $i$-th user and the H-AP. However, the nonlinear one involves an exponential function. So the optimization scheme is different using different energy harvest model. Although the nonlinear energy harvest model is more accurate and closer to reality, we aim to show the superiority of the proposed TR-WPCN, so we use the linear energy harvest model for simplicity.

Based on the above analysis, the signal-to-interference and noise ratio (SINR) of the $i$-th user in the uplink is as follows:

$$
\operatorname{SINR}_{i}=\frac{P_{i} g_{i}^{H} R_{i}^{(0)} g_{i}}{P_{i} g_{i}^{H} \widehat{R}_{i} g_{i}+\sum_{k \neq i} P_{k} g_{i}^{H} R_{k} g_{i}+\sigma^{2}},
$$

wherein $R_{i}^{(0)}=H_{L}^{(i) H} H_{L}^{(i)}, R_{k}=H_{k}^{H} H_{k}$, the superscript $(\bullet)^{H}$ is conjugate transpose, $H_{L}^{(i)}$ is the $L$-th row vector of $H_{i}$, and $\widehat{R}_{i}=H_{i}^{H} H_{i}-R_{i}^{(0)}$. In equation (10), $P_{i} g_{i}^{H} \widehat{R}_{i} g_{i}$ and $\sum_{k \neq i} P_{k} g_{i}^{H} R_{k} g_{i}$ represent ISI and IUI, respectively. To simplify the calculation, we assume that the variance of the different channel noises is the same.

Then, the uplink throughput of the $i$-th user can be obtained as follows:

$$
R_{i}=\tau^{U} \log _{2}\left(1+\operatorname{SINR}_{i}\right)
$$

According to equation (11), the system throughput can be derived as follows:

$$
R=\sum_{N} R_{i}, \quad i=1, \ldots, N
$$

\section{Time Allocation and Optimization}

According to the proposed transmission protocol, the time used for uplink wireless information transmission $\tau^{U} \leq 1-\tau^{D}-\tau^{\mathrm{tr}}$, so the uplink throughput of the $i$-th user $R_{i} \leq\left(1-\tau^{D}-\tau^{\mathrm{tr}}\right) \log _{2}\left(1+\mathrm{SINR}_{i}\right)$. Only consider the scenario the system throughput is maximum, that is, all the time after transmitting the probe signal is used to transmit the signal uplink and $R_{i}=\left(1-\tau^{D}-\tau^{\mathrm{tr}}\right) \log _{2}\left(1+\operatorname{SINR}_{i}\right)$. So the time used for DL energy transmission $\tau^{D}$ and the time used for $\operatorname{TR} \tau^{\mathrm{tr}}$ are analyzed as shown in Figure 3. It can be seen that the system throughput has a convex function with the change of $\tau^{D}$ and the system throughput has a maximum value, while the system throughput is a monotonously decreasing function with the change of $\tau^{\mathrm{tr}}$. To make the system throughput large enough, the time for time reversal should

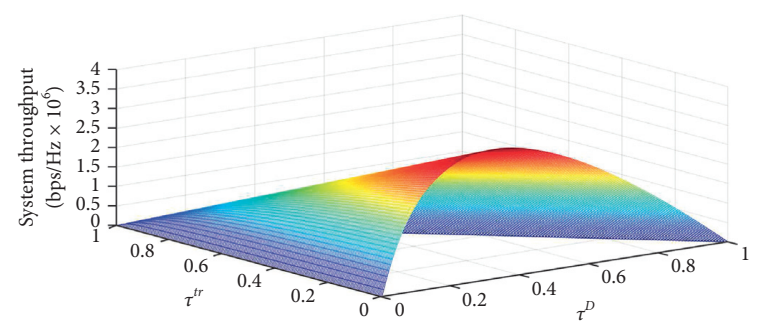

Figure 3: The relationship between system throughput and the time allocation scheme when $\tau^{U}=1-\tau^{D}-\tau^{\text {tr }}$ with $N=2, L=8$, $P_{A}=1 \mathrm{~mW}, \sigma^{2}=-50 \mathrm{dBm} / \mathrm{Hz}$, and $\eta=0.8$.

be as small as possible. However, the distance between users and H-AP should also be taken into account to ensure that the farthest user from the H-AP can complete the probe signal transmission phase of TR. Assume that the user's farthest distance from the HAP is $d$, so $\tau^{\mathrm{tr}}=d / v_{0}$, and $v_{0}$ is the information transmission rate.

To guarantee the QoS in the WPCN system, the SINR of each user must meet a threshold value. Therefore, under the premise of meeting the QoS needs of users, the total throughput of the system is maximized as shown in the following equation:

$$
\begin{gathered}
\max _{\tau^{D}, \tau^{\mathrm{rr}}, \tau^{U}} \sum_{N} \tau^{U} \log _{2}\left(1+\mathrm{SINR}_{i}\right), \\
\tau^{D} \geq 0 \\
\text { s.t. } \quad \tau^{U} \geq 0 \\
P_{A} \geq 0 \\
\tau^{D}+\tau^{\mathrm{tr}}+\tau^{U} \leq 1 \\
\operatorname{SINR}_{i} \geq \varepsilon \\
\tau^{\operatorname{tr}} \geq \frac{d}{v_{0}}
\end{gathered}
$$

where $\varepsilon$ is the minimum threshold value of the user's signalto-interference ratio. Further refine the objective function equation (13) as follows:

$$
R_{i}^{\prime}=\sum_{N} \tau^{U} \log _{2}\left(1+\frac{A \tau^{D}}{B \tau^{D}+C \tau^{U}}\right),
$$

where $\quad A=\eta_{i} \xi P_{A}\left|\widetilde{h}_{i}\right|^{2} g_{i}^{H} R_{i}^{(0)} g_{i}, \quad B=\eta_{i} \xi P_{A}\left|\widetilde{h}_{i}\right|^{2} g_{i}^{H} \widehat{R}_{i} g_{i}+$ $\sum_{k \neq i} \eta_{i} \xi P_{A}\left|\widetilde{h}_{i}\right|^{2} g_{i}^{H} R_{k} g_{i}$, and $C=\sigma^{2}$ for facilitation.

Theorem 1. The optimal value for the time-reversal phase is $\left(\tau^{t r}\right)=d / v_{0}$.

Proof. Fix $\tau^{U}$ and $\tau^{D}$ and solve the optimal solution of $\tau^{\mathrm{tr}}$, and then, the objective function becomes 


$$
\begin{gathered}
\max _{\tau^{\mathrm{tr}}} \sum_{N}\left(1-\tau^{\mathrm{tr}}-\tau^{D}\right) \log _{2}\left(1+\operatorname{SINR}_{i}\right), \\
\sum_{N}\left(1-\tau^{\mathrm{tr}}-\tau^{D}\right) \log _{2}\left(1+\operatorname{SINR}_{i}\right) \\
\geq \sum_{N} \tau^{U} \log _{2}\left(1+\operatorname{SINR}_{i}\right), \\
\tau^{\mathrm{tr}} \leq 1-\tau^{U}-\tau^{D}, \\
\tau^{\operatorname{tr}} \geq \frac{d}{v_{0}}
\end{gathered}
$$

In equation (15), $R\left(\tau^{\mathrm{tr}}\right)$ is a monotonically decreasing function about $\tau^{\mathrm{tr}}$; therefore, $\left(\tau^{\mathrm{tr}}\right)^{*}=d / v_{0}$.

Theorem 1 is proved.

After finding the optimized $\tau^{\mathrm{tr}}$, the objective function becomes

$$
\begin{gathered}
\max _{\tau^{D}, \tau^{U}} \sum_{N} \tau^{U} \log _{2}\left(1+\operatorname{SINR}_{i}\right), \\
\\
\text { s.t. } \quad \tau^{D} \geq 0 \\
\\
{ }^{U} \geq 0 \\
P_{A} \geq 0
\end{gathered}
$$

$$
\begin{gathered}
\tau^{D}+\tau^{U} \leq 1-\left(\tau^{\mathrm{tr}}\right)^{*} \\
\operatorname{SINR}_{i} \geq \varepsilon
\end{gathered}
$$

The objective function equation (16) is a concave function concerning $\tau^{U}$ and $\tau^{D}$ [23-25]. Fix $\tau^{D}$, and then, the objective function becomes

$$
\begin{aligned}
& \max _{\tau^{U}} \sum_{N} \tau^{U} \log _{2}\left(1+\operatorname{SINR}_{i}\right), \\
& \tau^{D} \geq 0, \\
& \text { s.t. } \quad P_{A} \geq 0, \\
& \tau^{U} \leq 1-\left(\tau^{\mathrm{tr}}\right)^{*}, \\
& \log _{2}\left(1+\operatorname{SINR}_{i}\right) \geq \log _{2}(1+\varepsilon) .
\end{aligned}
$$

For constraints (17a) and (17b), introduce the Lagrange multipliers $\lambda_{0}$ and $\lambda_{i}(i=1, \ldots, N)$, respectively; let $\lambda=\left[\lambda_{0}, \ldots, \lambda_{N}\right]$, then the Lagrangian function of the problem is as shown in the following equation:

$$
L\left(\tau^{U}, \lambda\right)=\sum_{N} \tau^{U} \log _{2}\left(1+\frac{A \tau^{D}}{B \tau^{D}+C \tau^{U}}\right)-\lambda_{0}\left[\tau^{U}+\left(\tau^{\mathrm{tr}}\right)^{*}+\tau^{D}-1\right]-\sum_{N} \lambda_{i}\left[\log _{2}(1+\varepsilon)-\log _{2}\left(1+\frac{A \tau^{D}}{B \tau^{D}+C \tau^{U}}\right)\right]
$$

The dual function of problem (18) is

$$
g(\lambda)=\max _{\tau^{U}} L\left(\tau^{U}, \lambda\right)
$$

$$
\min _{\lambda \geq 0} g(\lambda)
$$

The KKT (Karush-Kuhn-Tucher) conditions given in (21) are necessary:

and the dual problem becomes

$$
\frac{\partial L\left(\tau^{U}, \lambda\right)}{\partial \tau^{U}}=\log _{2}\left(1+\frac{A \tau^{D}}{B \tau^{D}+C \tau^{U}}\right)-\lambda_{0}+\sum_{i=1}^{N}\left(\tau^{U}+\lambda_{i}\right) \frac{1}{1+\left(A \tau^{D} /\left(B \tau^{D}+C \tau^{U}\right)\right)} \frac{-A C \tau^{D}}{\left(B \tau^{D}+C \tau^{U}\right)^{2}}=0
$$

Further simplification is shown in the following equation:

$$
\begin{aligned}
\frac{\partial L\left(\tau^{U}, \lambda\right)}{\partial \tau^{U}}= & \sum_{i=1}^{N} \log _{2}\left(1+\frac{A \tau^{D}}{B \tau^{D}+C \tau^{U}}\right)-\sum_{i=1}^{N}\left(\frac{C \lambda_{i}}{A \tau^{D}}+\frac{C}{A}\right) \frac{1}{1+\left(A \tau^{D} /\left(B \tau^{D}+C \tau^{U}\right)\right)} \\
& -\sum_{i=1}^{N}\left(\frac{C \lambda_{i}}{A \tau^{D}}+\frac{C}{A}\right)\left(1+\frac{A \tau^{D}}{B \tau^{D}+C \tau^{U}}\right)-2 \sum_{i=1}^{N}\left(-\frac{C \lambda_{i}}{A \tau^{D}}+\frac{C}{A}\right)-\lambda_{0} .
\end{aligned}
$$

Therefore, the solution of $\left(\partial L / \partial \tau^{U}\right)=0$ is the solution of the function $f\left(x_{i}\right)=\sum_{i=1}^{N} \log _{2} x_{i}+\sum_{i=1}^{N} p_{i}\left(1 / x_{i}\right)-\sum_{i=1}^{N} p_{i} x_{i}-$ $q=0$ which is $x_{i}{ }^{*}$, where $p_{i}=\left(C / A \tau^{D}\right) \lambda_{i}-\left(C / A_{i}\right), x_{i}=1+$
$\left(A \tau^{D} /\left(B \tau^{D}+C \tau^{U}\right)\right) \quad$ and $\quad q=2 \sum_{i=1}^{N}\left(\left(C / A \tau^{D}\right) \lambda_{i}+\right.$ $(C / A))-\lambda_{0}$, and the optimal solution for the uplink information transmission time can be solved as 
(1) Calculate $\left(\tau^{\mathrm{tr}}\right)^{*}$ with given $d$ and $v_{0}$;

(2) Initialize $t^{\text {low }}=0$ and $t^{\text {up }}=1-\left(\tau^{\text {tr }}\right)^{*}$ such that $t^{\text {opt }}$ lies in $\left[t^{\text {low }}, t^{\text {up }}\right]$;

(3) Repeat

(1) $t=\left(t^{\text {low }}+t^{\text {up }}\right) / 2$;

(2) Initialize $\lambda \geq 0$;

(3) Compute $g(\lambda)$ using (19). If $g(\lambda)>0$, go to step 4; if $g(\lambda)<0$, update $g(\lambda)$ using (23)-(24);

(4) Compute $\left(\tau^{U}\right)^{*}$ using (23);

(5) Substitute $\left(\tau^{U}\right)^{*}$ into (24) and compute $R(t)$ using (24);

(6) Check $R(t)>R\left(t^{\text {up }}\right)$. If it is satisfied, let $t^{\text {up }}=t$; otherwise, go to step 5;

(7) Check $R(t)<R\left(t^{\text {low }}\right)$. If it is satisfied, let $t^{\text {low }}=t$;

(4) Until $t^{\text {up }}-t^{\text {low }} \leq \mu$, where $\mu>0$ is a given error tolerance.

Algorithm 1: Time allocation and optimization algorithm.

$$
\left(\tau^{U}\right)^{*}=\frac{A \tau^{D}-B\left(x_{i}^{*}-1\right) \tau^{D}}{C\left(x_{i}^{*}-1\right)} .
$$

Then, a variabletis introduced, where $0 \leq t \leq 1-\left(\tau^{\mathrm{tr}}\right)^{*}$. Let $\tau^{U}+\tau^{D}=t$, at this point, the objective function becomes a function of $t$ :

$$
R(t)=\left(\tau^{U}\right)^{*} \log _{2}\left(1+\frac{A\left(t-\left(\tau^{U}\right)^{*}\right)}{B\left(t-\left(\tau^{U}\right)^{*}\right)+C\left(\tau^{U}\right)^{*}}\right) .
$$

Then, we can update $\lambda$ using subgradient-based algorithms and the subgradient factor is shown as

$$
\nabla \lambda_{i}=\log _{2}\left(1+\frac{A \tau^{D}}{B \tau^{D}+C \tau^{U}}\right)-\log _{2}(1+\varepsilon), \quad 1 \leq i \leq N,
$$

where $\nabla \lambda_{i}$ is the increment of subgradient in the process of iteration and the dual function is updated by

$$
\lambda_{i}^{(k+1)}=\left[\lambda_{i}^{(k)}+c \lambda_{i}^{(k+1)}\right]_{0}^{+}, \quad 1 \leq i \leq N,
$$

where $\varsigma$ is the step size of Lagrange multiplier during iteration. Based on the above foundation, combined with the dichotomy method, time allocation and optimization algorithm for TR-WPCN is proposed, as shown in Algorithm 1.

\section{Numerical Result}

In this section, we compare the proposed scheme with the conventional schemes proposed in $[5,14]$. And the impact on system performance of the proposed scheme under different conditions is analyzed.

In the simulation process, the channel obeys the Rayleigh distribution and the system bandwidth is $1.4 \mathrm{MHz}$. For each user, the energy utilization rate is assumed to be $\eta=0.8$ and the path loss factor between users is $\gamma_{1}=\cdots=\gamma_{i}=2$. Finally, we define the power spectral density of the noise $\sigma^{2}=-50 \mathrm{dBm} / \mathrm{Hz}$.

Figure 4 shows the relationship between the average user throughput and the number of users in different scenarios, where the transmit power is $1 \mathrm{~mW}$. By comparing with the other two schemes proposed in $[5,14]$, the impact of

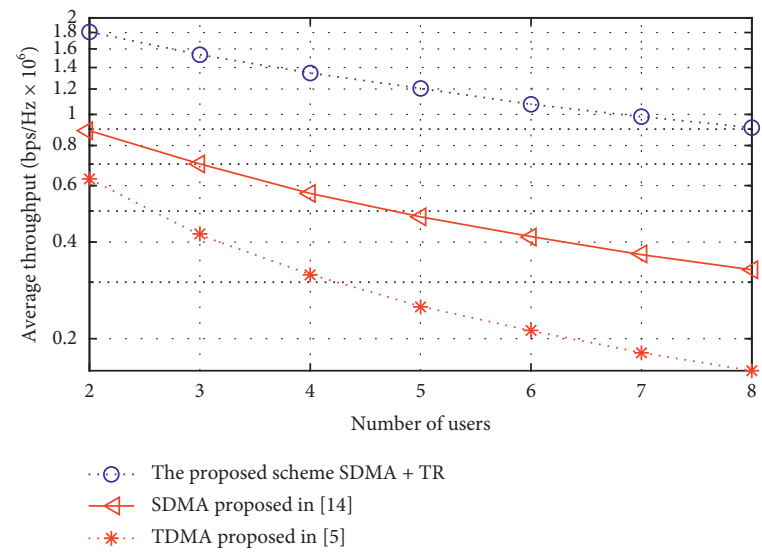

Figure 4: Comparison of average user throughput about the proposed scheme and the traditional schemes with $L=8, N=2$, $\sigma^{2}=-50 \mathrm{dBm} / \mathrm{Hz}$, and $\eta=0.8$.

introducing TR on the system throughput can be intuitively analyzed. Assume that the time $\tau^{D}$ used for DL wireless energy transmission in this paper is equal to the SDMA scheme proposed in [14] and the TDMA scheme proposed in [5], that is, all schemes are equally on $\tau^{D}$. Suppose all users use the same time to transmit information uplink via the TDMA scheme proposed in [5]. From Figure 5, the proposed scheme can effectively improve system throughput. Compared with the scheme proposed in [14], the average throughput increases by $51.9 \%$ which is twice that of the scheme proposed in [5]. Since the transmission power is constant, the average throughput of users decreases as the number of users increases in all schemes. However, with the increase in the number of users, the reduction of the average throughput of the users in the proposed scheme is smaller than other schemes. Compared with the method of using only SDMA in [14], TR-WPCN can effectively suppress IUI and ISI during uplink information transmission, thus improving the system throughput and stability. In the scheme using TDMA in [5], there is no IUI, but the UL transmission consumes too much time, so the spectrum utilization is lower and the system throughput declined relatively. In summary, compared with the traditional time allocation scheme, the proposed scheme can effectively improve the 


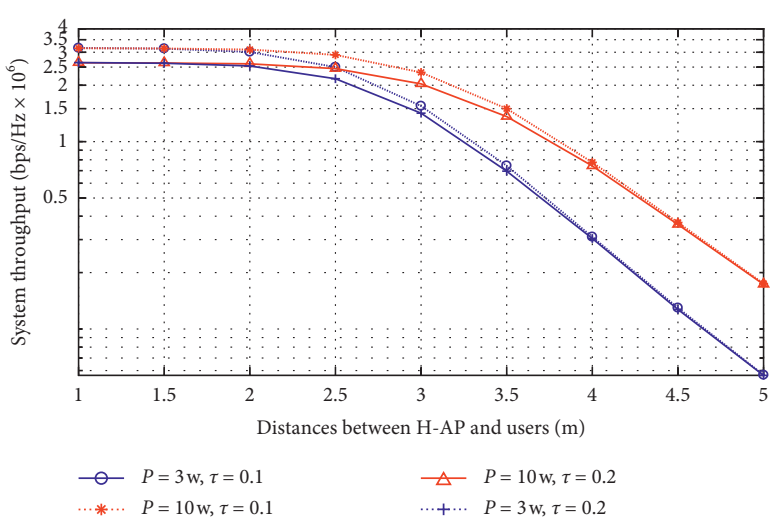

FIgURE 5: The relationship between the distance from user to the $\mathrm{H}$ $\mathrm{AP}$ and the system throughput with $L=8, \quad N=2$, $\sigma^{2}=-50 \mathrm{dBm} / \mathrm{Hz}$, and $\eta=0.8$.

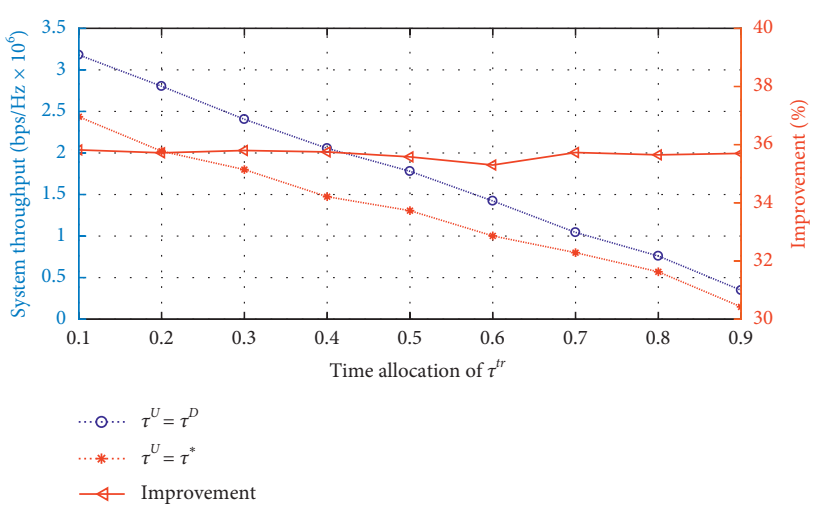

Figure 6: The relationship between probe time and the system throughput with $L=8, N=2, \sigma^{2}=-50 \mathrm{dBm} / \mathrm{Hz}$, and $\eta=0.8$.

average throughput of users and the stability of the system is stronger.

In Figure 5, we show the sum throughput versus the distance from the users to the H-AP. We set four cases to show the effect of the transmission power and $\tau^{\text {tr }}$ on the system throughput. The horizontal axis is in meters. In Figure 5, as the distance from the users to the H-AP increases, the system throughput gradually decreases, which is consistent with the "double near" problem described above. Moreover, the smaller the transmission power is, the greater the reduction of the system throughput there is. When the time ratio of the TR phase is larger, the throughput of the system is smaller and this is consistent with the previous research content. So the time used for TR should be as small as possible but no less than $d / v_{0}$. Therefore, the system throughput is inversely proportional to the distance between the user to the H-AP and the time spent in the time-reversal phase.

In Figure 6, system throughput is plotted versus the rate of $\tau^{\text {tr }}$ cost in a block transmission time. The blue line in Figure 6 indicates the throughput of the system when the optimization algorithm finds the optimal time allocation scheme $\tau^{U}=\tau^{*}$, such as when $\tau^{\mathrm{tr}}=0.1$ and $\tau^{U}=\tau^{*}=0.289$; the red line indicates the throughput of the system when
$\tau^{U}=\tau^{D}$, such as if $\tau^{\mathrm{tr}}=0.1$, then $\tau^{U}=\tau^{*}=0.45$. Both lines correspond to the left $Y$-axis. From Figure 6 , as the time spent in the probing phase increases, the system throughput gradually decreases. Moreover, the solid line with a triangle corresponds to the $Y$-axis on the right side, which indicates the increment of the system throughput compared to the optimal time allocation scheme with the scheme when $\tau^{U}=\tau^{*}$. It shows that the system throughput is improved by about $35.7 \%$ compared with the $\tau^{U}=\tau^{*}$ scheme. This further proves that the system throughput is inversely proportional to the time spent in the time-reversal phase.

\section{Conclusion}

In this paper, we studied a WPCN composed of an H-AP and $N$ users. Aiming at the uplink transmission interference problem of multiuser WPCN system, we combined with time reversal to focus the transmission signal and restrain interference. Moreover, a time allocation algorithm is proposed to maximize the system throughput. We explained how to solve the system throughput maximization problem while explicitly considering channel state information and the distance between the H-AP and users. We simplified the optimization by processing the original problem as an optimization problem corresponding to each variable and solve the problems in stages. The simulation results prove that the proposed scheme can effectively suppress interference and improve system performance from the aspects of average user throughput and total system throughput. And the average throughput increases by $51.9 \%$ compared with that reported in [14]; meanwhile, the average throughput is twice of that proposed in [5]. The proposed TR-WPCN has broad application prospects and can be used in wireless sensor networks. Future research can allocate and optimize resources for multiantenna $\mathrm{H}-\mathrm{AP}$ and multiuser complex multicell environments and vehicle networking environments.

\section{Data Availability}

The data used to support the findings of this study are available from the corresponding author upon request.

\section{Conflicts of Interest}

The authors declare that they have no conflicts of interest.

\section{Acknowledgments}

This work was supported by the Natural Science Foundation of China (NSFC) grant funded by the China Government (61771084, 61102062, and 61271260) and also funded by the Natural Science Foundation of the Science and Technology Committee in Chongqing (cstc2015jcyjA40050).

\section{References}

[1] G. Dong, H. Zhang, and D. Yuan, "Downlink achievable rate of massive MIMO enabled SWIPT systems over rician 
channels," IEEE Communications Letters, vol. 22, no. 3, pp. 578-581, 2018.

[2] X. Chen, X. Wang, and X. Chen, "Energy-efficient optimization for wireless information and power transfer in largescale MIMO systems employing energy beamforming," IEEE Wireless Communications Letters, vol. 2, no. 6, pp. 667-670, 2013.

[3] J. Nithya and P. Latha, "Energy harvest and transfer technology in wireless networks," in Proceedings of World Conference on Futuristic Trends in Research and Innovation for Social Welfare, pp. 1-4, Coimbatore, India, March 2016.

[4] M. U. Kim and H. J. Yang, "Min-SINR maximization with DL SWIPT and UL WPCN in multi-antenna interference networks," IEEE Wireless Communications Letters, vol. 6, no. 3, pp. 318-321, 2017.

[5] H. Ju and R. Zhang, "Throughput maximization in wireless powered communication networks," IEEE Transactions on Wireless Communications, vol. 13, no. 1, pp. 418-428, 2014.

[6] A. Biason and M. Zorzi, "Transmission policies in wireless powered communication networks with energy cooperation," in Proceedings of the 24th European Signal Processing Conference (EUSIPCO), pp. 592-596, Budapest, Hungary, August 2016.

[7] Z. Chu, F. Zhou, Z. Zhu, R. Q. Hu, and P. Xiao, "Wireless powered sensor networks for Internet of Things: maximum throughput and optimal power allocation," IEEE Internet of Things Journal, vol. 5, no. 1, pp. 310-321, 2018.

[8] M. A. Abd-Elmagid, T. ElBatt, and K. G. Seddik, "Optimization of wireless powered communication networks with heterogeneous nodes," in Proceedings of the IEEE Global Communications Conference (GLOBECOM), pp. 1-7, San Diego, CA, USA, December 2015.

[9] M. A. Abd-Elmagid, T. ElBatt, and K. G. Seddik, "Optimization of energy-constrained wireless powered communication networks with heterogeneous nodes," Wireless Networks, vol. 25, no. 2, pp. 713-730, 2019.

[10] M. S. H. Abad and O. Ercetin, "Optimal finite horizon sensing for wirelessly powered devices," IEEE Access, vol. 7, pp. 131473-131487, 2019.

[11] H. Ju and R. Zhang, "Optimal resource allocation in fullduplex wireless-powered communication network," IEEE Transactions on Communications, vol. 62, no. 10, pp. 35283540, 2014.

[12] X. Kang, C. K. Ho, and S. Sun, "Full-duplex wireless-powered communication network with energy causality," IEEE Transactions on Wireless Communications, vol. 14, no. 10, pp. 5539-5551, 2015.

[13] M. A. Abd-Elmagid, A. Biason, T. ElBatt, K. G. Seddik, and M. Zorzi, "On optimal policies in full-duplex wireless powered communication networks," in Proceedings of the 14th International Symposium on Modeling and Optimization in Mobile, Ad Hoc, and Wireless Networks (WiOpt), pp. 1-7, Tempe, AZ, USA, May 2016.

[14] L. Liu, R. Zhang, and K.-C. Chua, "Multi-antenna wireless powered communication with energy beamforming," IEEE Transactions on Communications, vol. 62 , no. 12, pp. 43494361, 2014.

[15] M. A. Abd-Elmagid, A. Biason, T. ElBatt, K. G. Seddik, and M. Zorzi, "Non-orthogonal multiple access schemes in wireless powered communication networks," in Proceedings of the IEEE International Conference on Communications (ICC), pp. 1-6, Paris, France, May 2017.

[16] M. M. N. Aboelwafa, M. A. Abd-Elmagid, A. Biason, K. G. Seddik, T. ElBatt, and M. Zorzi, "Towards optimal resource allocation in wireless powered communication networks with non-orthogonal multiple access," Ad Hoc Networks, vol. 85, pp. 1-10, 2019.

[17] H. Ma, B. Wang, Y. Chen, and K. J. Ray Liu, "Time-reversal tunneling effects for cloud radio access network," IEEE Transactions on Wireless Communications, vol. 15, no. 4, pp. 3030-3043, 2016.

[18] H. V. Tran, G. Kaddoum, H. Tran, D. Tran, and D. Ha, “Time reversal SWIPT networks with an active eavesdropper: SERenergy region analysis," in Proceedings Of IEEE VTC-Fall, pp. 1-5, Montreal, Canada, September 2017.

[19] Y. Chen, F. Han, Y.-H. Yang et al., "Time-reversal wireless paradigm for green Internet of Things: an overview," IEEE Internet of Things Journal, vol. 1, no. 1, pp. 81-98, 2014.

[20] H. Y. Huang, Research on propagation characteristics of time reversal electromagnetic wave, Ph.D. thesis, University of Electronic Science and Technology of China, Chengdu, China, 2014.

[21] G. D. Ge, Super-resolution characteristics timereversed electromagnetic wave and its application, Ph.D. thesis, University of Electronic Science and Technology of China, Chengdu, China, 2012.

[22] P. Blomgren, P. Kyritsi, A. D. Kim, and G. Papanicolaou, "Spatial focusing and intersymbol interference in multipleinput-single-output time reversal communication systems," IEEE Journal of Oceanic Engineering, vol. 33, no. 3, pp. 341$355,2008$.

[23] B. Stephen, V. Lieven, and B. Stephen, "Convex optimization," IEEE Transactions on Automatic Control, vol. 51, no. 11, p. 1859, 2006.

[24] H. Lim and T. Hwang, "User-centric energy efficiency optimization for MISO wireless powered communications," IEEE Transactions on Wireless Communications, vol. 18, no. 2, pp. 864-878, 2019.

[25] Z. Alessio and J. Eduard, Energy Efficiency in Wireless Networks via Fractional Programming Theory, Now Publishers Inc., Boston, MA, USA, 2015. 